\title{
Desastres naturais e saúde: uma análise da situação do Brasil
}

\author{
Natural disasters and health: an analysis of the situation in Brazil
}

\author{
Carlos Machado de Freitas ${ }^{1}$ \\ Diego Ricardo Xavier Silva ${ }^{2}$ \\ Aderita Ricarda Martins de Sena ${ }^{3}$ \\ Eliane Lima Silva ${ }^{3}$ \\ Luiz Belino Ferreira Sales ${ }^{3}$ \\ Mauren Lopes de Carvalho ${ }^{1}$ \\ Maíra Lopes Mazoto ${ }^{4}$ \\ Christovam Barcellos ${ }^{2}$ \\ André Monteiro Costa ${ }^{5}$ \\ Mara Lúcia Carneiro Oliveira ${ }^{6}$ \\ Carlos Corvalán ${ }^{6}$
}

${ }^{1}$ Centro de Estudos e

Pesquisas em Emergências e Desastres em Saúde (Cepedes), Escola Nacional de Saúde Pública, Fundação Oswaldo Cruz (Fiocruz). Avenida Brasil 4036/916, Manguinhos. 21.040-361 Rio de Janeiro RJ Brasil. carlosmf@ensp.fiocruz.br

${ }^{2}$ Observatório Nacional Clima e Saúde, Instituto de Informação e Comunicação em Saúde (Icict), Fundação Oswaldo Cruz (Fiocruz).

${ }^{3}$ Secretaria de Vigilância em Saúde, Ministério da Saúde.

${ }^{4}$ Instituto de Estudos em Saúde Coletiva,

Universidade Federal do Rio de Janeiro.

${ }^{5}$ Centro de Pesquisa Aggeu Magalhães, Fiocruz.

${ }^{6}$ Organização Pan-

Americana da Saúde,

Organização Mundial da

Saúde (OPAS/OMS), Brasil.
Abstract Natural disasters are still insufficiently studied and understood within the scope of public health in this country, with impacts in the short and long term. The scope of this article is to analyze the relationship between disasters and their impact on health based on disaster data recorded in the country. The methodology involved the systematization of data and information contained in the Brazilian Atlas of Natural Disasters 19912010 and directly from the National Department of Civil Defense (NSCD). Disasters were organized into four categories of events (meteorological; hydrological; climatological; geophysical/ geological) and for each of the latter, the data for morbidity, mortality and exposure of those affected were examined, revealing different types of impacts. Three categories of disasters stood out: the hydrological events showed higher percentages of mortality, morbidity and exposure; climatological events had higher percentages of incidents and people affected; the geophysical/geological events had a higher average of exposure and deaths per event. Lastly, a more active participation of the health sector in the post-2015 global political agenda is proposed, particularly events related to sustainable development, climate change and disaster risk reduction.

Key words Disasters, Natural disasters, Health situation, Environmental health
Resumo Os desastres naturais ainda são pouco pesquisados e compreendidos no âmbito da Saúde Coletiva no país, com impactos do curto ao longo prazos. O objetivo deste artigo é, a partir de dados sobre desastres registrados no país, analisar a inter-relação entre esses eventos e seus impactos sobre a saúde. A metodologia envolveu a sistematização de dados e informações contidos no Atlas Brasileiro dos Desastres Naturais 1991-2010 e diretamente na Secretária Nacional de Defesa Civil (SNDC). Os desastres foram organizados em quatro categorias de eventos (meteorológicos; hidrológicos; climatológicos; geofísicos/geológicos) e, para cada uma das mesmas, foram explorados os dados de afetados, morbidade, mortalidade e expostos, demonstrando diferentes tipos de impactos. Três categorias de desastres se destacaram: os eventos hidrológicos apresentaram maiores percentuais de mortalidade, morbidade e expostos; os climatológicos maiores percentuais de ocorrências e afetados; os geofísicos/geológicos maior média de expostos e óbitos por evento. Ao final propõe-se uma participação mais ativa do setor saúde na agenda política global pós-2015, particularmente as relacionadas ao desenvolvimento sustentável, mudanças climáticas e redução de riscos de desastres.

Palavras-chave Desastres, Desastres naturais, Situação de saúde, Saúde ambiental 


\section{Introdução}

Sinais de que os desastres naturais podem se tornar cada vez mais frequentes e/ou mais graves vêm ocorrendo através de diversos eventos. Em 2005 e 2010, secas atingiram o estado do Amazonas, em uma das regiões de maior disponbilidade hídrica do país e do planeta. Em 2009 e 2012, este mesmo estado sofreu inundações graduais que superaram os níveis históricos. Em 2004, o ciclone Catarina atingiu o litoral norte do Rio Grande do Sul e sul de Santa Catarina causando danos na área urbana e rural, concentradas numa faixa de aproximadamente 100 km. Em 2008, Santa Catarina foi afetada por fortes inundações bruscas, assim como Alagoas e Pernambuco em 2010. Em 2011, a Região Serrana do Rio de Janeiro foi atingida pelo mais grave desastre em termos de óbitos imediatos (quase mil), envolvendo inundações e deslizamentos. Em 2013, três em cada quatro municípios do Nordeste estavam em situação de emergência por conta de uma seca iniciada em 2010 e considerada uma das mais longas dos últimos 50 anos.

Cada um destes eventos exemplificam um tema: os desastres naturais, ainda muito pouco pesquisados e compreendidos no âmbito da Saúde Coletiva no país. Os impactos destes desastres sobre a saúde das populações não se restringem somente aos de curto prazo e registrados nos períodos imediatamente após os mesmos, mas envolvem também efeitos de médio e longos prazos. O objetivo deste artigo é, a partir de dados sobre desastres naturais no país, analisar a interrelação entre estes eventos e seus impactos sobre a saúde. Consideramos que este tipo de análise é fundamental para avançarmos tanto no desenvolvimento de políticas públicas, como para as ações de prevenção, mitigação, preparação, resposta e reabilitação para reduzir o impacto dos desastres sobre a saúde.

\section{O que são os desastres naturais}

Um desastre natural resulta da combinação de quatro fatores importantes para a Saúde Coletiva, que são: 1) a ocorrência de uma ameaça natural; 2) uma população exposta; 3 ) as condições de vulnerabilidade social e ambiental desta população; 4) insuficientes capacidades ou medidas para reduzir os potenciais riscos e os danos à saúde da população ${ }^{1,2}$. Esses eventos envolvem simultaneamente processos naturais e sociais, que impactam a sociedade a partir do padrão de interação entre os eventos de origem natural e a organização social ${ }^{3}$.
As ameaças se relacionam à qualidade dos eventos físicos que podem ser gerados pela dinâmica da natureza. Esses eventos possuem qualidades específicas por sua localização geográfica, magnitude, intensidade, frequência e probabilidade ${ }^{1,4}$. As ameaças podem ser individuais, combinadas ou sequenciais em suas origens e consequências.

A exposição é um conceito chave na saúde ambiental, pois é o que permite estabelecer as possíveis interelações entre a população - ou determinados grupos populacionais (crianças, idosos, mulheres etc.) presentes em um determinado lugar $-\mathrm{e}$ as situações ambientais alteradas por eventos disparadores que são as ameaças naturais, tais como chuvas fortes, inundações bruscas ou graduais, secas ou estiagens, ou por condições latentes de degradação ambiental ${ }^{2}$.

As condições de vulnerabilidade resultam de processos sociais e mudanças ambientais que denominamos de vulnerabilidade socioambiental, pois combinam: a) os processos sociais que resultam na precariedade das condições de vida e proteção social (trabalho, renda, saúde e educação, assim como aspectos ligados a infraestrutura, como habitações saudáveis e seguras, estradas, saneamento, entre outros), que tornam determinados grupos populacionais (por exemplo, idosos, mulheres e crianças), principalmente entre os mais pobres, vulneráveis aos desastres; b) as mudanças ambientais resultantes da degradação ambiental (áreas de proteção ambiental ocupadas, desmatamento de encostas, ocupação desordenada do solo urbano, precariedade de sistemas de drenagem e resíduos sólidos, poluição de águas, solos e atmosfera, entre outros), que tornam determinadas áreas mais vulneráveis frente à ocorrência de ameaças e seus eventos subsequentes.

As condições de vulnerabilidades estabelecem territórios críticos em diferentes escalas e em diferentes temporalidades, o que coloca a questão dos desastres como um problema essencialmente socioambiental, desmistificando a ideia de um evento imponderável ou apenas de origem natural. Os territórios críticos são assim as áreas reais, onde as contradições sociais apontadas como geradoras de vulnerabilidade estão estabelecidas ${ }^{5}$. Desta maneira, os territórios críticos são áreas delimitáveis, onde se concentram grupos sociais vulneráveis e se produzem condições ambientais favoráveis ao agravamento das vulnerabilidades.

Além disto, é importante observar que o desastre por suas próprias características e dinâmica não só atualiza uma situação de risco e vulnerabilidade existente, como cria novos cenários 
de riscos e vulnerabilidades, a depender das condições ambientais, sociais e sanitárias do território. Essas condições de riscos e vulnerabilidades exigem políticas contínuas e integradas voltadas à redução de risco de desastres, pois envolvem as próprias capacidades para a prevenção de riscos presentes e futuros, bem como políticas para a preparação, respostas, reabilitação, e reconstrução de sociedades ou comunidades afetadas.

\section{Desastres naturais e seus potenciais impactos sobre a saúde}

Estudos sobre impactos dos desastres naturais na saúde humana destacam que estes afetam as populações de forma desigual e de maneiras diferentes, direta e indiretamente, com efeitos que variam de curto a longo prazos, a depender das características do evento e da vulnerabilidade socioambiental do território ${ }^{6}$.

Dessa forma, os impactos dos desastres naturais sobre a saúde podem ocorrer em tempos diferentes, caracterizando-se em períodos que variam entre horas a anos. No curto prazo de tempo, entre horas a alguns dias, se produzem a maior parte dos registros de feridos leves e graves e mortalidade, incluindo como resposta as ações de resgate e urgência. Um segundo momento, se dá no período entre dias a semanas, caracterizando-se pela ocorrência de algumas doenças transmissíveis, a exemplo da leptospirose e doenças diarreicas, podendo agravar quadros de doenças não transmissíveis em pacientes crônicos, como, por exemplo, a hipertensão. Nesta fase, é importante iniciar ações de vigilância, controle e prevenção de doenças, assim como, a reabilitação dos serviços necessários à assistência à saúde e outros serviços essenciais, como o abastecimento de água e alimentos, por exemplo. Num espaço maior de tempo, entre meses e anos, os impactos na saúde se relacionam às doenças não transmissíveis, especialmente, os transtornos psicossociais e comportamentais, as doenças cardiovasculares, desnutrição e a intensificação de doenças crônicas.

A Figura 1, adaptada de Redmond ${ }^{7}$ retrata estes períodos de respostas frente aos impactos sobre a saúde provocados pelos desastres naturais.

Os impactos diretos e indiretos que os desastres naturais podem causar na saúde humana e nos determinantes de saúde podem ser mais bem entendidos quando exploradas as interrelações entre os tipos de eventos e suas consequências no ambiente e na sociedade afetada

O Quadro 1, elaborado por Freitas e Ximenes $^{8}$ para situações de inundações e adaptado para as situações de seca e estiagem ${ }^{9,10}$, de modo a cobrir os desastres predominantes no Brasil, abordam os impactos que esses tipos de desastres provocam sobre o ambiente e sobre as infraestruturas de serviços, economia e sociedade local do território afetado.

Em relação às consequências ambientais, podemos considerar que estes tipos de eventos, comprometem os serviços de saneamento ambiental, a quantidade e a qualidade da água, o solo e oferta de alimentos, bem como alterações

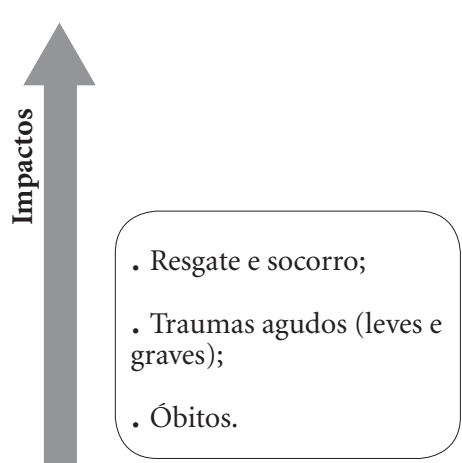

Horas - Dias

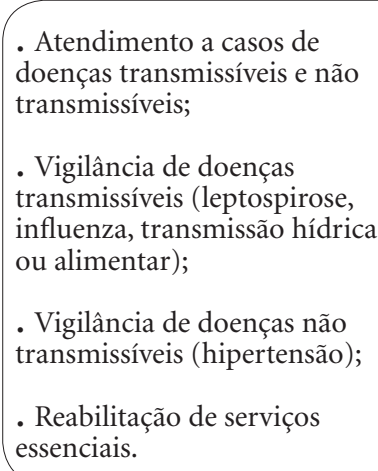

- Atendimento a casos de doenças transmissíveis e não transmissíveis;

- Vigilância de doenças transmissíveis (leptospirose, influenza, transmissão hídrica ou alimentar);

- Vigilância de doenças não transmissíveis (hipertensão);

- Reabilitação de serviços essenciais.

- Atenção e vigilância das doenças crônicas;

- Vigilância de doenças não transmissíveis (cardiovasculares; psicossociais);

- Reabilitação e reconstrução
Meses - Anos

Escala temporal

Figura 1. Respostas do setor saúde aos desastres em diferentes tempos. 
Quadro 1. Consequências ambientais e socioecnômicas dos desastres provocados por inundações/enxurradas/ alagamentos e seca/estiagem.

\begin{tabular}{|c|c|c|}
\hline & Consequências ambientais & Consequências socioeconômicas \\
\hline 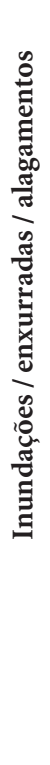 & $\begin{array}{l}\text {. Contaminação biológica e química da água } \\
\text { para consumo humano, alimentos e solo } \\
\text {. Comprometimento da rede e fontes } \\
\text { alternativas de abastecimento de água } \\
\text {. Comprometimento da rede de serviço de } \\
\text { coleta e tratamento de esgoto } \\
\text {. Comprometimento dos serviços de coleta e } \\
\text { disposição do lixo } \\
\text { - Alteração nos ciclos dos vetores, hospedeiros } \\
\text { e reservatórios de doenças e nas formas de } \\
\text { exposições ambientais dos humanos }\end{array}$ & $\begin{array}{l}\text {. Interrupção total ou parcial de pontes, ruas e estradas } \\
\text { por inundação ou destruição } \\
\text {. Rompimento de diques de contenção } \\
\text {. Rompimento de tanques de combustíveis } \\
\text {. Interrupção total ou parcial do fornecimento } \\
\text { de serviços de água, eletricidade, gás, transporte e } \\
\text { comunicação } \\
\text { - Interrupção total ou parcial do funcionamento de } \\
\text { escolas, comércio, serviços funerários, serviços de saúde } \\
\text { e outros } \\
\text { - Comprometimento total ou parcial das atividades } \\
\text { agrícolas e pecuárias } \\
\text {. Prejuízos econômicos pela destruição total ou parcial } \\
\text { de propriedades, casas e construções } \\
\text {. Prejuízos econômicos pela destruição total ou parcial } \\
\text { das fontes de renda e trabalho } \\
\text {. Perdas de bens pessoais e de valor sentimental } \\
\text {. Rompimento ou fortalecimento da amizade, } \\
\text { cooperação e laços afetivos entre os membros de uma } \\
\text { comunidade afetada }\end{array}$ \\
\hline 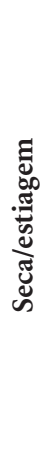 & $\begin{array}{l}\text {. Contaminação da água para consumo } \\
\text { humano, alimentos e solo } \\
\text {. Comprometimento da rede e fontes } \\
\text { alternativas de abastecimento de água } \\
\text {. Intrusão de água salgada em suprimentos de } \\
\text { água doce subterrânea } \\
\text {. Contaminação do ar por poeira e partículas } \\
\text { oriundas de incêndios, de florações de } \\
\text { cianobactérias e de toxinas acumuladas no solo } \\
\text { - Alteração nos ciclos dos vetores, hospedeiros } \\
\text { e reservatórios de doenças e nas formas de } \\
\text { exposições ambientais dos humanos }\end{array}$ & $\begin{array}{l}\text {. Interrupção total ou parcial do fornecimento de água } \\
\text {. Comprometimento da quantidade e qualidade de água } \\
\text { para consumo humano } \\
\text {. Comprometimento total ou parcial das atividades } \\
\text { agrícolas, pecuárias e pesqueiras } \\
\text {. Comprometimento da quantidade e qualidade de } \\
\text { alimentos } \\
\text {. Prejuízos econômicos pela destruição total ou parcial } \\
\text { das fontes de renda e trabalho } \\
\text {. Perdas de bens pessoais e de valor sentimental devido } \\
\text { à necessidade de migrar e à incapacidade de suprir a } \\
\text { família com elementos essenciais à vida }\end{array}$ \\
\hline
\end{tabular}

nos ciclos de vetores, hospedeiros e reservatórios de doenças. No que se refere às consequências sobre a infraestrutura, serviços, economia e sociedade local, os elementos básicos que dão suporte às condições de vida e à saúde, entre estes, os próprios serviços de saúde, podem ficar comprometidos.

Além dessas consequências que as inundações, secas e estiagem podem causar no ambiente e sobre a infraestrutura, serviços, economia e sociedade local, devem ser consideradas também as características próprias dos diferentes tipos de ameaças que podem resultar em múltiplos efeitos, conforme demonstrados na Figura 2, que sistematiza os efeitos considerados mais comuns sobre a saúde humana decorrentes de desastres naturais, por tipologia de evento.
Conforme demonstram o Quadro 1, combinado com as Figuras 1 e 2, os efeitos sobre a saúde humana decorrentes dos impactos pelos diferentes tipos de desastres, não só podem provocar múltiplos efeitos sobre a saúde humana no curto, médio e longo prazos, como também efeitos que se sobrepõem, constituindo um grande desafio para os sistemas nacionais e locais de atenção e vigilância em saúde.

Isto significa que as políticas, recursos e ações requeridos para reduzir os riscos e os impactos devem incluir não só o período dos impactos imediatos identificados dias após o desastre, mas também os períodos de semanas e meses, dada a importância de recuperação e reconstrução das condições de vida e saúde em situação melhor do que a anterior ao desastre. Em caso de conse- 


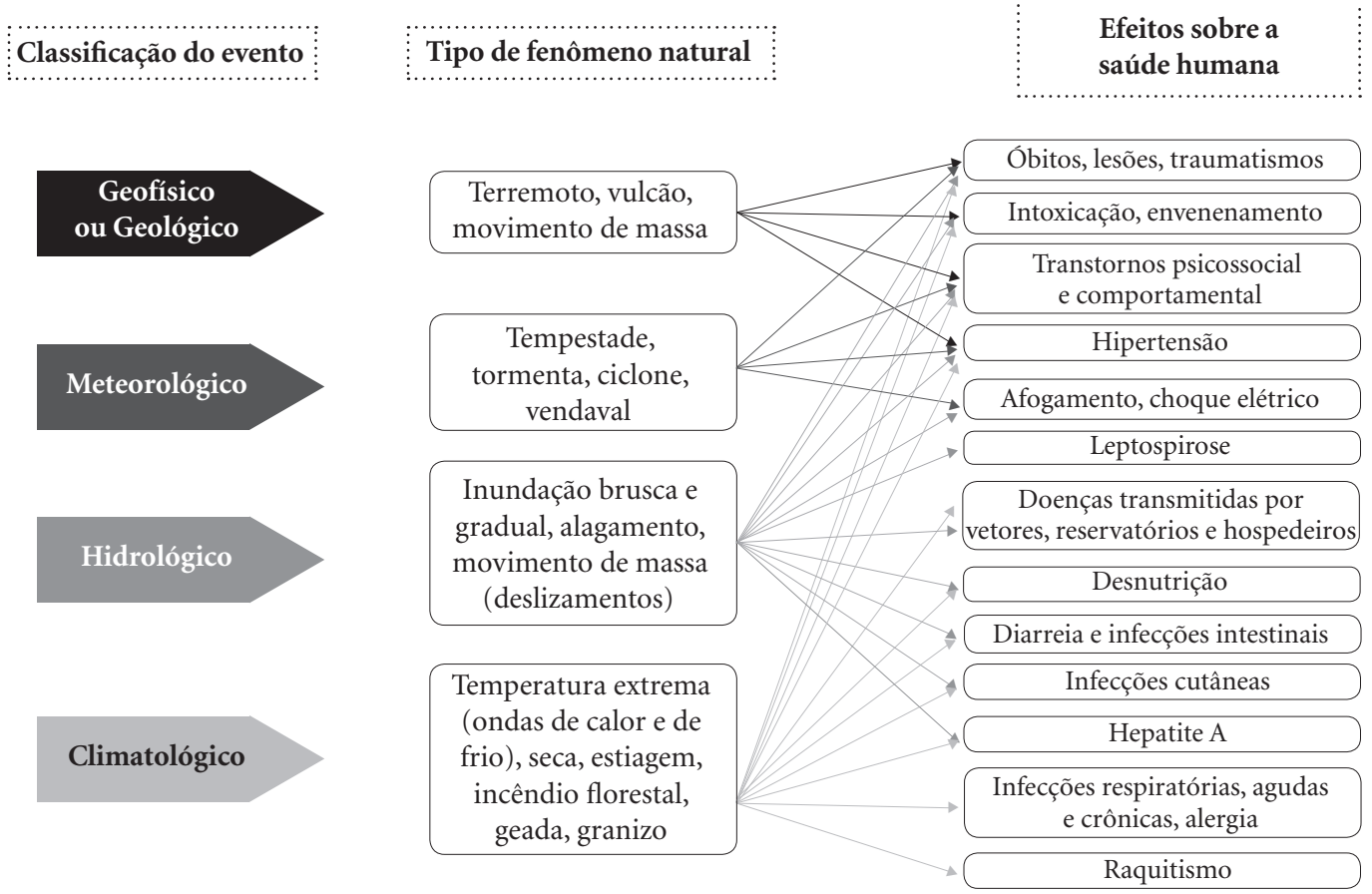

Figura 2. Tipos de fenômenos naturais e seus efeitos sobre a saúde humana.

quências mais graves, é recomendável que essas ações envolvam os anos suficientes para recuperar as condições de saúde da população e infraestrutura dos serviços.

\section{Metodologia para análise da situação dos desastres naturais no Brasil}

Para a análise da situação dos desastres naturais no Brasil e seus riscos para a saúde, utilizamos dois conjuntos de dados e informações.

O primeiro, já contido no Atlas Brasileiro dos Desastres Naturais 1991-2010, volume Brasil, publicado pelo Centro Universitário de Estudos e Pesquisas Sobre Desastres da Universidade Federal de Santa Catarina ${ }^{11}$, que envolveu os registros da Secretária Nacional de Defesa Civil (SNDC), mas também os das Secretarias Estaduais de Defesa Civil.

Os dados deste Atlas tiveram como principais fontes os documentos que eram elaborados primariamente no nível municipal da defesa civil e enviados para o estadual e depois federal, sendo estes a Notificação Preliminar de Desastre (NOPRED) e o Avaliação de Danos (AVADAN). A partir daqui será referido através da sigla ABDN 1991-2010. O segundo, através da coleta e sistematização dos dados sobre decretos de situação de emergência (SE) e estado de calamidade pública (ECP), registrados somente na SNDC no período 2003-2012. Ambas as fontes são importantes para analisar os eventos, mas possuem como limitações os diferentes níveis de qualidade no preenchimento das informações e por abordar somente os impactos imediatos e registrados nos primeiros cinco dias após um desastre.

Em ambas as fontes, os dados foram classificados segundo as seguintes ameaças naturais: estiagem e seca; inundação brusca e alagamentos; inundação gradual; granizo; geada; vendaval e/ ou ciclone; tornado; incêndios florestais; movimento de massa; erosão linear; erosão fluvial; erosão marinha.

Para exemplificar essas ameaças, tomamos como exemplos as categorias de desastres que se encontram organizadas nas bases de dados internacionais sobre desastres naturais, como SIGMA, NatCatSERVICE e Centre for Research on the Epidemiology of Disasters (CRED) $)^{12}$, sendo estas:

. Eventos geológicos ou geofísicos: envolvem os processos erosivos, de movimentação de massa e deslizamentos resultantes de processos geológicos ou fenômenos geofísicos.

. Eventos meteorológicos: envolvem os processos que resultam em fenômenos como ciclones tropicais e extratropicais, tornados e vendavais. 
. Eventos hidrológicos: envolvem os processos que resultam em alagamentos, enchentes, inundações graduais e bruscas.

- Eventos climatológicos: envolvem os processos relacionados à estiagem e seca, queimadas e incêndios florestais, chuvas de granizo, geadas e ondas de frio e de calor.

Para análise dos dados do Atlas, consideramos a classificação adotada pelo mesmo para afetados (que envolve qualquer pessoa que tenha sido atingida ou prejudicada por um desastre, direta e indiretamente) e mortalidade. Para morbidade, agrupamos os dados de enfermos gravemente feridos e levemente feridos. Para expostos, agrupamos os dados de todos os diretamente afetados (deslocados, desabrigados e desalojados), embora não constassem nos registros de mortalidade e morbidade imediatas ao desastre. Este agrupamento permitiu identificar os níveis de gravidade para os eventos. Quanto aos limites dos dados de morbidade e mortalidade é que se referem somente ao período imediatamente pósdesastre registrados pelos órgãos de Defesa Civil.

Os dados coletados diretamente nos registros da SNDC permitiram elaborar os mapas de 1 a 5 na Figura 3, identificando o conjunto de municípios de maior vulnerabilidade a esses eventos, mas com o limite de não dispor de dados sobre morbidade, mortalidade e expostos.

\section{Resultados}

\section{Os Desastres no Brasil - Da Situação à Exposição}

Se por um lado os desastres para que ocorram necessitam de um evento natural, como fenômeno disparador, que concretiza o cenário de exposição aos riscos atuais, por outro, alteram a situação ambiental nas áreas em que ocorrem e criam novos cenários de exposição aos riscos.

Conforme podemos observar na Tabela 1 , em 20 anos foram registrados no Brasil 31.909 desastres afetando a vida de mais de 96 milhões de pessoas e obrigando que mais de 6 milhões deixassem seus lares. Mesmo restrito ao período imediatamente pós-desastre (primeiros dias) e não contabilizando os impactos de médio e longo prazos sobre a mortalidade e morbidade, foram registrados cerca 3,5 mil óbitos e $490 \mathrm{mil}$ pessoas ficaram enfermas ou feridas.

Do total de eventos, $57,8 \%$ foram eventos climatológicos, predominando seca e estiagem. Estes eventos foram os que mais afetaram a população no Brasil, com quase 50 milhões de afetados e correspondendo a mais da metade do total. Os eventos hidrológicos, principalmente inundações bruscas, correspondem a $32,7 \%$ do total, com quase 39 milhões de afetados. Em termos de impactos diretos sobre a população, são os eventos hidrológicos os que apresentam maior número de mortalidade, morbidade e diretamente expostos. Em termos de letalidade (óbitos por evento) chama a atenção os eventos geofísicos ou geológicos, com uma média de 2 óbitos por evento, representando, em média, 13 vezes mais chances de óbitos do que nos casos dos eventos hidrológicos, que tiveram o maior número total de óbitos.

Conforme podemos ver na Figura 3 (Mapa 1) contendo os decretos de SE e ECP por municípios, entre 2003 e 2012, há três grandes núcleos de municípios vulneráveis aos desastres naturais no país. O primeiro, nos estados do Nordeste, principalmente de Alagoas ao Ceará. O segundo, no Norte de Minas Gerais e Sul da Bahia. O terceiro, nos estados do Sul do país, principalmente Santa Catarina e Rio Grande do Sul. Os desastres que predominam nestes núcleos de municípios vulneráveis serão detalhados na análise das categorias de desastres a seguir.

\section{Eventos Hidrológicos}

Conforme podemos observar na Tabela 1, os eventos hidrológicos (inundações graduais e bruscas) corresponderam a $32,7 \%$ dos eventos ocorridos no Brasil, $40,3 \%$ dos afetados, $44,8 \%$ da mortalidade, $63,5 \%$ da morbidade e $67,5 \%$ dos expostos, principalmente desalojados e desabrigados. Tais eventos afetam as diferentes regiões do país de maneira distinta, tanto do ponto de vista da frequência quanto da distribuição ao longo do ano.

Os dados do ABDN 1991-2010 revelam que no período houve um aumento na frequência das inundações bruscas no Brasil, com picos em 1997, 2004, 2009 e 2010. Quanto à distribuição no ano, janeiro e fevereiro foram os meses de maior ocorrência no país. O maior número de inundações bruscas foi registrado na região Sul $(36,5 \%)$, seguida da região Sudeste $(30 \%)$ e Nordeste $(24,5 \%)$. A região Sudeste foi a mais atingida em número de afetados e concentrou mais da metade (52\%) de todos os óbitos ocorridos por inundações bruscas no Brasil.

Os registros de inundações graduais no Brasil aumentaram significativamente da década de 1990 para a década de 2000, havendo picos nos anos de 2004 e 2009. As inundações graduais concentram-se nas regiões Sudeste $(31,5 \%)$, Nordeste $(28 \%)$ e Sul $(22,6 \%)$, sendo a região Norte também bastante afetada, com 11,7\% dos registros. Nesses tipos de eventos, a região Nordeste 


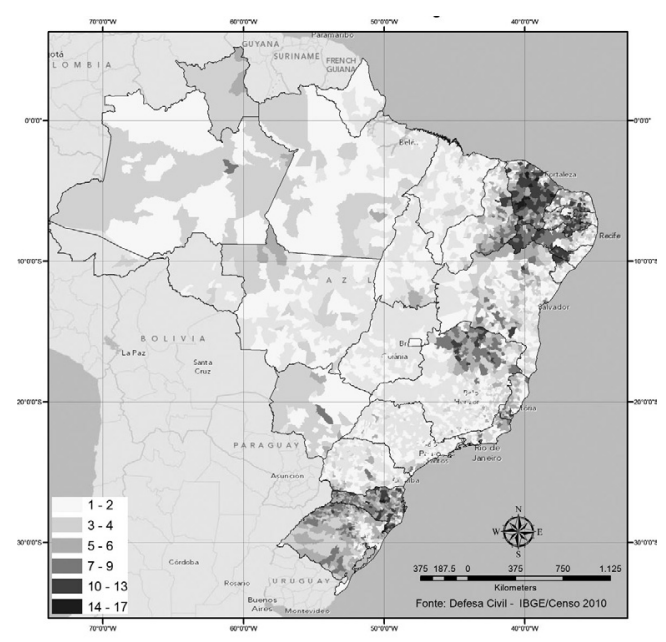

1) Todos os eventos

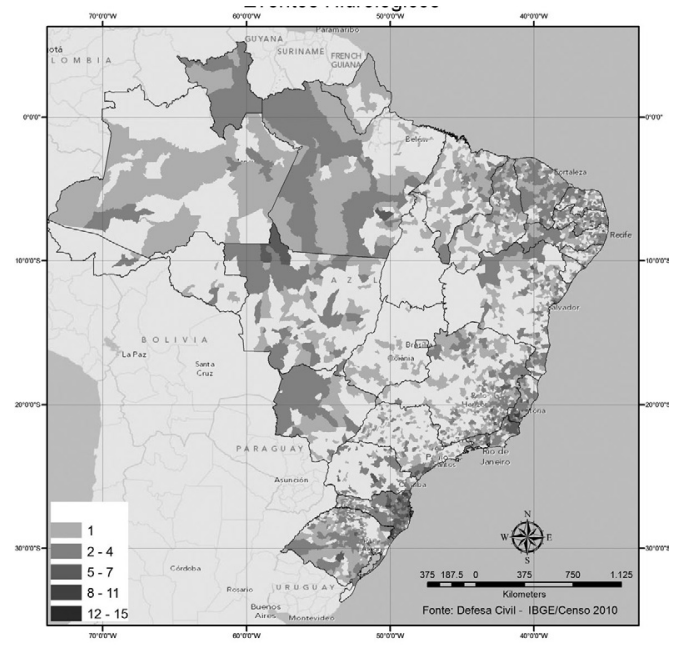

2) Eventos hidrológicos



4) Eventos metereológicos

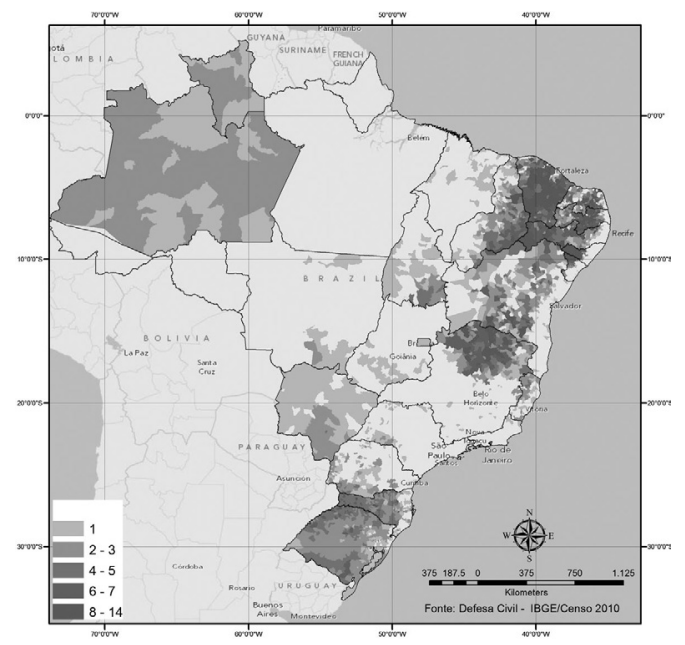

3) Eventos climatológicos

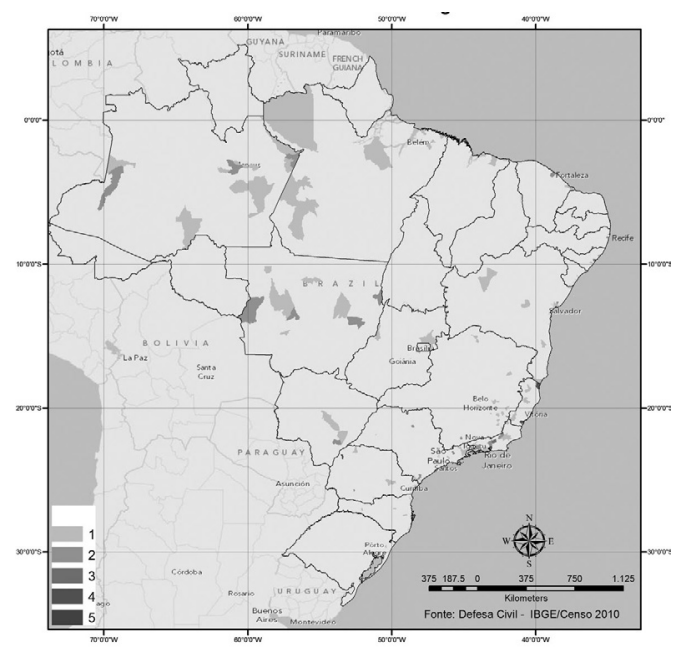

5) Eventos geofísicos ou geológicos

Figura 3. Mapas dos decretos de situação de emergência e decretos de estado de calamidade pública, Brasil, 2003-2012. 
Tabela 1. Registro de Desastres Naturais no Brasil, 1991-2010.

\begin{tabular}{lrrrcr}
\hline Tipos de desastres & $\begin{array}{c}\text { Total dos } \\
\text { eventos }\end{array}$ & Afetados & Mortalidade & $\begin{array}{c}\text { Morbidade } \\
\text { (enfermos e feridos } \\
\text { leves e graves) }\end{array}$ & $\begin{array}{c}\text { Diretamente expostos } \\
\text { (deslocados desabrigados } \\
\text { desalojados) }\end{array}$ \\
\hline Hidrológicos & 10.444 & 38.836 .257 & 1.567 & 309.529 & 4.176 .851 \\
Climatológico & 18.450 & 49.868 .081 & 273 & 167.582 & 1.554 .450 \\
Meteorológico & 2.290 & 4.120 .439 & 161 & 4.917 & 276.847 \\
Geológico/Geofísico & 725 & 3.544 .059 & 1.403 & 5.530 & 173.259 \\
Total & 31.909 & 96.368 .836 & 3.404 & 487.558 & 6.181 .407 \\
\hline
\end{tabular}

Fonte: CEPED UFSC, 2012.

reuniu o maior número de afetados $(41 \%)$ e a região Sudeste, o maior número de óbitos (59,5\%).

Tendo como referência a Tabela 1 , podemos constatar também que os desastres hidrológicos são os que apresentam maior média de morbidade $(n=29)$ e expostos $(n=399)$ por evento. É cerca do dobro da proporção de morbidade $(\mathrm{n}=$ $15)$ e expostos $(n=193)$ por eventos para todas as categorias de desastres.

Conforme podemos ver na Figura 3 (Mapa 2), os estados das Regiões Sul e Sudeste do país foram os que concentraram o maior número de municípios com o maior número de decretos para eventos hidrológicos.

\section{Eventos Climatológicos}

De acordo com a Tabela 1, os eventos climatológicos (estiagem e seca, incêndios florestais, chuvas de granizo, geadas e ondas de frio e de calor) correspondem a 57,8\% dos eventos registrados no Brasil, 52\% dos afetados, $8 \%$ da mortalidade, $34,5 \%$ da morbidade e $25 \%$ dos expostos diretos, devendo observar que estes eventos concentram $64,5 \%$ do total da população deslocada, ou seja aquela que por motivos de desastres é obrigada a migrar.

Segundo dados do ABDN 1991-2010, dentre os eventos climatológicos, a estiagem e a seca representam, aproximadamente $97 \%$ do total dos mesmos. Seca e estiagem tiveram seus picos de eventos na década de 2000, que concentrou 75\% das ocorrências registradas no período analisado. A distribuição desses eventos ao longo do período analisado (20 anos) demonstra que a região Nordeste apresentou aproximadamente $60 \%$ dos registros, com destaque para os meses de maio, março, outubro e abril como os de maior número de registros. Minas Gerais é também um estado bastante afetado por estiagens e secas, principalmente na parte Norte do mesmo, que integra o semiárido brasileiro. A Região Nordeste foi a que concentrou a maior parte dos impac- tos humanos por seca e estiagem, com $72 \%$ dos afetados, cerca de $80 \%$ dos expostos e $67 \%$ dos óbitos. Em relação à morbidade, a região Norte concentrou $56 \%$ do total do país, seguida pela região Nordeste com 29\%.

Conforme podemos observar na Figura 3 (Mapa 3), dois grandes núcleos de municípios concentram o maior número de decretos de desastres relacionados aos eventos climatológicos. O primeiro encontra-se na Região do Semiárido, envolvendo desde o Norte de Minas Gerais até um conjunto de estados da Região Nordeste (exceto o Maranhão, que não faz parte do semiárido). O segundo núcleo encontra-se na Região Sul do país, principalmente nos estados de SC e RS. Não menos importante aparecem na Região Norte os estados do AM e RR.

\section{Eventos Meteorológicos}

$\mathrm{Na}$ Tabela 1 podemos verificar que os desastres meteorológicos (raios, ciclones tropicais e extratropicais, tormenta, tornados e vendavais) representaram $7 \%$ do total das ocorrências de desastres naturais no Brasil, $4 \%$ dos afetados, $5 \%$ da mortalidade, $1 \%$ da morbidade e $4,5 \%$ dos expostos.

De acordo com o ABDN 1991-2010, a distribuição dos 2.290 eventos (41 tornados e 2.249 vendavais e ciclones) ao longo dos 20 anos demonstra que a região Sul concentrou cerca de $80 \%$ dos eventos (839 municípios atingidos), $80 \%$ dos afetados e $83 \%$ dos óbitos. O maior número de eventos ocorreu no estado do Rio Grande do Sul, que foi o estado mais atingido, com $29 \%$ das ocorrências registradas no país. Os meses de picos foram setembro, outubro e novembro para vendavais e ciclones; e janeiro, setembro e outubro para tornados, com $71 \%$ destes eventos específicos ocorrendo em Santa Catarina.

Conforme podemos verificar na Figura 3 (Mapa 4), a maior parte destes eventos se concentrou na Região Sul do país, principalmente SC e RS. 


\section{Eventos Geológicos ou Geofísicos}

Conforme podemos ver na Tabela 1, os desastres geofísicos ou geológicos (terremoto, vulcão, movimento de massa) corresponderam a $1,4 \%$ dos desastres ocorridos no país, $4 \%$ dos afetados, $41 \%$ da mortalidade, $1 \%$ da morbidade e $3 \%$ dos expostos. Sua característica mais importante é a alta letalidade.

De acordo com o ABDN 1991-2010, 81,7\% das ocorrências de movimentos de massa foram registradas na região Sudeste. A região Sul é a segunda mais atingida, contabilizando $12,3 \%$ dos casos de desastres por movimentos de massa.

No Brasil, muitos dos movimentos de massa estão diretamente relacionados às chuvas fortes. Os meses de novembro e dezembro e os primeiros meses do ano, de janeiro a abril, concentram a maior parte desses eventos, com maior pico de ocorrências em janeiro.

Por outro lado, o mapa dos decretos de SE e ECP, Figura 3 (Mapa 5) mostra que os desastres geofísicos ou geológicos como um todo apresentam uma distribuição mais dispersa pelo país. De acordo com o mapa, a maior concentração dos registros se deu no estado do Amazonas e na Região Sudeste, principalmente nos estados do Espírito Santo e Rio de Janeiro.

É importante destacar que, se por um lado os eventos geológicos correspondem aos desastres menos frequentes no Brasil, por outro são os que apresentam as maiores médias de pessoas afetadas $(\mathrm{n}=4.888)$ e óbitos $(\mathrm{n}=1,9)$ por evento, sendo a média geral de afetados por evento 3.020 e de mortalidade 0,1 .

\section{Discussão}

Ao apresentar os resultados do ABDN 1991-2010 sintetizados na Tabela 1 e os dados da SNDC nos mapas da Figura 3, organizados em categorias de eventos, procuramos contribuir para uma aproximação entre o tema dos desastres e a Saúde Coletiva. Desastres não se realizam sem a ocorrência de ameaças naturais, bem como impactos sobre a saúde não se realizam sem estas e a exposição, que pode ser agravada em condições de vulnerabilidade socioambiental.

Simultaneamente cabe lembrar que quase a totalidade dos desastres registrados no Brasil, tanto nos decretos SE e ECP quanto nos dados levantamentos na SNDC, tem origem direta ou indireta em processos climáticos, que envolvem os ventos, chuvas e estiagens que, por sua vez, podem gerar deslizamentos, inundações, enchentes e secas. Tanto a frequência como os efeitos destes eventos extremos tendem a crescer com o processo de mudanças ambientais e climáticas globais, o que inclui o aquecimento global.

É na confluência dos processos globais, tanto os socioeconômicos quanto os climáticos, como dos processos nacionais e locais de desenvolvimento, que os desastres se realizam.

Em relação aos eventos específicos, os hidrológicos, conforme podemos observar na Tabela 1 , foram os que apresentaram maiores médias de morbidade e diretamente expostos por evento, 29 e 399 respectivamente. Porém, é importante distinguir as inundações bruscas das graduais, pois ainda que apresentem impactos comuns, possuem dinâmicas distintas.

Nas regiões Sul, Sudeste e Nordeste observamos uma maior frequência das inundações bruscas e resultantes de chuvas prolongadas ou intensas afetando as populações que ocupam as margens de rios. Como exemplos citamos as inundações de 2008 em SC, que resultaram em 110 óbitos e 121 mil diretamente expostos, entre desalojados e desabrigados ${ }^{13}$, e as de AL/PE em 2010, que resultaram em 56 óbitos e 178 mil diretamente expostos ${ }^{14,15}$. Muitas dessas inundações ocorrem rapidamente e, em alguns casos, as chuvas que contribuíram para as mesmas também provocam deslizamentos de terras combinando dois tipos de desastres, como foi o caso da Região Serrana em 2011, que resultou em 918 óbitos e mais de 31 mil diretamente expostos ${ }^{16}$. Em todos estes eventos, além dos impactos diretos sobre a saúde, os impactos sobre a infraestrutura, habitações e condições de vida certamente resultaram em impactos indiretos sobre as condições de saúde não devidamente avaliados.

De acordo com dados da Estratégia Internacional de Redução de Riscos de Desastres (EIRD $)^{17}$, inundações quando combinadas com deslizamentos podem resultar em 6 vezes mais óbitos do que quando ocorrem isoladamente. $\mathrm{O}$ caso da Região Serrana no RJ, quando comparado com os de SC em 2008 e AL/PE em 2010, aponta para um cenário mais grave. Se consideramos o indicador óbitos por diretamente expostos, temos para cada mil desalojados/desabrigados 0,3 óbitos em AL/PE, 0,9 em SC e 29,0 na Região Serrana/RJ, o que é uma variação de 32 à 96 mais óbitos para cada mil diretamente expostos, quando comparado este último desastre com os outros dois anteriores (AL/PE e SC).

$\mathrm{Na}$ região Norte do país, as inundações são graduais e fazem parte da dinâmica dos rios na região Amazônica. Em 2009 e 2012 duas inundações no AM alertam não só para a elevação do nível dos rios durante as cheias (atingiram níveis 
maiores do que os registrados até então, que havia sido em 1953), como o aumento da população exposta. Em 2012 uma inundação ainda maior que a de 2009 atingiu a região da capital amazonense. Em ambos os eventos cerca de 20 mil famílias vivendo em habitações com condições precárias, próximas a igarapés, sofreram com o avanço das águas sobre suas casas. Doenças como diarreias, leptospirose e dermatites, entre outras de veiculação hídrica, apresentaram surtos em toda a cidade $^{18}$. Mas não somente nas margens dos rios e igarapés a população tem sido exposta, pois em 2012 ocorreu também o caso do município de Anamã (AM), que localizado na confluência dos rios Solimões e Purus, em área de várzea, foi todo ele atingido e, a população de pouco mais de 11 mil habitantes, cerca de 8 mil, foi afetada e mais de 3 mil diretamente expostos ${ }^{19}$.

Mas, se a Região Amazônica é conhecida pela disponibilidade de recursos hídricos e podemos associar isto diretamente às inundações, importante observar que a mesma vem também sendo afetada pelas secas. No estado do Amazonas, 2005 e 2010 foram os anos das maiores secas já registradas, a primeira afetando mais de $167 \mathrm{mil}$ pessoas e a segunda cerca de 122 mil pessoas. Estas secas afetam diretamente as principais vias de transporte, isolando alguns municípios e comunidades, aumentando os riscos de agravos e doenças. Importante observar que se a região Norte concentrou apenas pouco mais de $1 \%$ dos eventos de seca e estiagem, em relação à morbidade, a região concentrou $56 \%$ do total do país.

Ainda em relação aos desastres climatológicos, seca e estiagem são os eventos de maior importância na região Nordeste e Norte de MG, na Região do Semiárido. O semiárido brasileiro possui aproximadamente 22 milhões de habitantes e engloba cerca de $20 \%$ dos municípios do país, sendo $90 \%$ destes de pequeno porte, onde encontram-se os mais baixos índices de Desenvolvimento Humano Municipal (em 2006 70\% dos municípios com o mais baixo IDH-M se encontravam no semiárido) e a maior parte (58\%) da população pobre do país ${ }^{20,21}$. Esta região, caracterizada por grande vulnerabilidade socioambiental, concentrou a maior parte dos impactos humanos em termos de afetados, expostos e óbitos por este tipo de desastre, particularmente em relação aos expostos. Importante destacar que estes tipos de eventos são os que resultam na maior parte da população deslocada, ou seja, aquela que não só perde sua habitação, mas migra para outro município.

Se no ABDN 1991-2010 os desastres hidrológicos (inundações) e climatológicos (princi- palmente secas e estiagens) respondem conjuntamente por mais de $90 \%$ dos eventos, dos afetados, da morbidade e dos diretamente expostos, são os desastres geofísicos/geológicos os que apresentam maior letalidade. Embora este tipo de desastre tenha correspondido à apenas 1,4\% dos desastres ocorridos no país, foi responsável por $40 \%$ da mortalidade. Tomando a Tabela 1 como referência, a letalidade de cada desastre geofísico/ geológico resultou em cerca de 1,9 óbitos, o que é 13 vezes maior do que os desastres hidrológicos ( 0,15 óbito por evento).

Como última categoria de eventos, temos os desastres meteorológicos, concentrados principalmente na região Sul do país (SC e RS). Embora responsáveis por cerca de 7\% dos eventos, não apresentam indicadores de gravidade significativos. Entretanto não podemos deixar de observar mudanças que podem estar se processando a partir de eventos como o Ciclone Catarina, que atingiu o litoral norte do Rio Grande do Sul e sul de Santa Catarina, resultando em 1 óbito, 518 lesionados e cerca de 33 mil diretamente expostos. Apesar da controvérsia em torno do mesmo, é um evento importante que alerta sobre a relação entre mudanças climáticas e eventos extremos.

Por fim, dois aspectos importantes para a discussão que estão na raiz do próprio conceito de desastres. Se até aqui privilegiamos a análise tomando como referência as ameaças naturais e categorias de eventos, deve-se considerar também as condições de vulnerabilidade e população exposta e impactos sobre a saúde.

Em relação às condições de vulnerabilidade, parte da mesma se relaciona como se deu no Brasil o processo de urbanização de sua população, consolidado ao longo da segunda metade do século XX e que, dissociado de um processo de planejamento e organização do espaço urbano, resultou na concentração de populações nas áreas urbanas, associada a incapacidade de suprir a necessidades desse adensamento demográfico. O reflexo mais forte dessa lógica foi à consolidação de grandes aglomerados subnormais, principalmente em áreas ambientalmente frágeis ou degradas (principalmente encostas de morros e margens de rios), sem infraestrutura urbana e ocupada, predominantemente, pela população de menor poder socioeconômico ${ }^{22}$, constituindo assim territórios críticos, mais vulneráveis aos desastres relacionados às inundações e deslizamentos (somam cerca de $40 \%$ dos desastres de acordo com a Tabela 1).

Seguindo a mesma lógica do desenvolvimento que propiciou este processo de urbanização e organização do espaço, é importante considerar 
a ausência de políticas de longo prazo integradas e contínuas para o enfrentamento da seca e estiagem (acesso à água em quantidade e qualidade, bem como desenvolvimento econômico local para ampliar a oferta de empregos e produção de alimentos), principalmente no semiárido brasileiro, mas também considerando a região Sul do país. Situação similar se aplica à região amazônica, onde o desenvolvimento no nível local das comunidades para reduzir os riscos da seca e uma melhor ocupação do espaço para reduzir os riscos das inundações são vitais.

Em relação às populações expostas e aos impactos sobre a saúde, é importante considerar que, embora os desastres tenham afetado cerca de 96 milhões de pessoas ao longo de 20 anos, em determinadas áreas e lugares, com maiores condições de vulnerabilidade, é muito provável que a mesma população tenha sido afetada pelo mesmo tipo de desastre ao longo dos anos. A vivência de repetidos desastres, com suas consequências ambientais, socioeconômicas e sanitárias contribuem para uma sobreposição de riscos à saúde para estas populações.

Se consideramos que a grande maioria, se não a totalidade dos registros de mortalidade e morbidade realizados pelas Defesas Civis, concentram-se no período imediatamente pós-desastre, isto significa que o total de 3.494 óbitos (média de 0,1 óbito por desastre) e 487.558 enfermos e feridos (média de 15 por desastre) representam apenas uma parte dos reais riscos e efeitos dos desastres sobre a saúde das populações no Brasil. O mesmo se aplica para os dados sobre os mais de 6 milhões diretamente expostos (deslocados, desabrigados e desalojados) aos riscos de doenças (média de 193 por desastre). Se levarmos em conta que os riscos e os efeitos sobre a saúde são diversos, e se expressarão em diferentes momentos da vida dessas populações, ações de vigilância estruturadas, não somente para o curto prazo, no período imediatamente pó-desastre, mas também envolvendo ações de médio e longo prazos, integradas com as ações de atenção à saúde, poderiam revelar um conjunto de impactos sobre a saúde ainda ocultos.

\section{Considerações finais}

No âmbito da Saúde Coletiva, como observa Castellanos $^{23}$, a situação de saúde corresponde a formas de concretização, no nível particular, dos processos mais gerais que caracterizam a estrutura e dinâmica de uma sociedade, em dado momento de sua história. Isto envolve por um lado, os processos sociais e econômicos (o nível de desenvolvimento das forças produtivas, as relações sociais, o modelo de desenvolvimento econômico e de sua inserção internacional, a organização do estado e suas relações políticas). Por outro lado, também os relacionados às mudanças da situação ambiental, resultantes destes processos e que desencadeiam desde alterações nos ciclos do clima e das águas, até amplos processos de degradação dos recursos naturais disponíveis. Assim, se chuvas fortes, inundações, estiagens, secas, deslizamentos e ciclones constituem fenômenos da natureza, os desastres são fenômenos sociais relacionados aos modelos de desenvolvimento social e econômico adotados.

A natureza interdisciplinar e intersetorial dos desastres necessariamente obriga a Saúde Coletiva a ter uma ampla visão sobre os mesmos, bem como a formular políticas e ações que atuem diretamente sobre seus determinantes socioambientais. O ano de 2014 é bastante propício para isto, se considerarmos que teremos as discussões sobre três agendas globais pós-2015: Objetivos do Milênio (ODM) subsituídos pelos Objetivos do Desenvolvimento Sustentável (ODS); um novo acordo sobre Mudança Climática (Pós Kyoto) e uma nova agenda internacional para enfrentamento dos desastres, substituindo o Marco de Ação de Hyogo, que também se encerra em 2015. É uma grande oportunidade para discutirmos o tema no país e formularmos uma agenda integrada para o enfrentamento dos desastres. E, esperamos, com este tipo de análise, contribuir para que o setor saúde avance no debate sobre este tema, não só estruturando uma melhor resposta para as ações de vigilância e atenção no curto prazo, mas também de reabilitação e recuperação das condições de saúde, bem como reconstrução da vida das populações expostas, além da formulação de políticas intersetoriais de prevenção dos mesmos.

\section{Colaboradores}

CM Freitas, DRX Silva, ARM Sena, EL Silva, LBF Sales, ML Carvalho, ML Mazoto, C Barcellos, AM Costa, MLC Oliveira e C Corvalán participaram igualmente de todas as etapas de elaboração do artigo. 


\section{Referências}

1. Narváez L, Lavell A, Ortega GP. La gestión del riesgo de desastres: un enfoque basado en procesos. San Isidro: Secretaría General de la Comunidad Andina; 2009.

2. Freitas CM, Carvalho ML, Ximenes EF, Arraes EF, Gomes JO. Vulnerabilidade socioambiental, redução do risco de desastres e construção da resiliência - lições do terremoto no Haiti e das chuvas fortes na Região Serrana, Brasil. Cien Saude Colet 2012; 17(6):1577-1586.

3. Mateddi MA, Butzke IC. A relação entre o social e o natural nas abordagens de hazards e de desastres. Ambiente \& Sociedade 2001; 4(9):1-22.

4. Estrategia Internacional para la Reducción de Desastres de las Naciones Unidas (EIRD/ONU). Marco de Acción de Hyogo 2005-2015. Aumento de la resiliencia de las naciones y las comunidades ante los desastres: Introducción al Marco de Acción de Hyogo. Genebra: EIRD; 2005.

5. Santos SM, Barcellos C, organizadores. Abordagens espaciais na saúde pública. Brasília: Fundação Oswaldo Cruz, Secretaria de Vigilância em Saúde do Ministério da Saúde; 2006.

6. Alderman K, Turner LR, Tong S. Floods and human health: A systematic review. Environment International 2014; 31(1):169-190.

7. Redmond $\mathrm{AD}$. $\mathrm{ABC}$ of conflict and disaster. BMJ 2005; 330:1259-1261.

8. Freitas CM, Ximenes EF. Enchentes e saúde pública: uma questão na literatura científica recente das causas, consequências e respostas para prevenção e mitigação. Cien Saude Colet 2012; 17(6):1601-1616.

9. World Health Organization, United Nations Convention to Combat Desertification (WHO \& UNCCD). Our Planet, Our Health, Our Future. Human health and the Rio Conventions: biological diversity, climate change and desertification. Geneva: WHO, UNCCD; 2012.

10. Centers for Disease Control and Prevention (CDC), U.S. Environmental Protection Agency, National Oceanic and Atmospheric Agency, American Water Works Association. 2010. When every drop counts: protecting public health during drought conditions - a guide for public health professionals. Atlanta: CDC, U.S. Department of Health and Human Services; 2010.

11. Universidade Federal de Santa Catarina. Centro Universitário de Estudos e Pesquisas sobre Desastres (UFSC-CEPED). Atlas brasileiro de desastres naturais 1991 a 2010: volume Brasil. Florianópolis: CEPED -UFSC; 2012.

12. Kron W, Steuer M, Löw P, Wirtz A. How to deal properly with a natural catastrophe database - analysis of flood losses. Nat. Hazards Earth Syst. Sci. 2012; 12:535-550.

13. Banco Mundial (BM). Avaliação de Perdas e Danos: Inundações Bruscas em Santa Catarina - Novembro de 2008. Brasília: BM; 2012.

14. Banco Mundial (BM). Avaliação de Perdas e Danos: Inundações Bruscas em Pernambuco - Junho de 2010. Brasília: BM; 2012.
15. Banco Mundial (BM). Avaliação de Perdas e Danos: Inundações Bruscas em Alagoas - Junho de 2010. Brasília: BM; 2012.

16. Banco Mundial (BM). Avaliação de Perdas e Danos: Inundações e Deslizamentos na Região Serrana do Rio de Janeiro - Janeiro de 2011. Brasília: BM; 2012.

17. United Nations Conference on Sustainable Development (UNCSD). Disaster Risk Reduction and Resilience Building. [documento da internet]. 2012 [acessado 2012 jan 12], [4 p.]. Disponível em: http://www. uncsd2012.org/rio20/index.php?page $=$ view\&nr $=$ 225\&type $=400 \&$ menu $=45$

18. Silva DX, Barcellos C, Bacuri R. Vulnerabilidade e efeitos das mudanças climáticas na saúde pública em Manaus. Relatório do projeto "Guidance for Conducting Assessments of Health Vulnerability and Public Health and Health Care Interventions to Address ClimateChange", financiado pela Organização Panamericana de Saúde (ContratoBR/CNT/0991502.001 de outubro de 2009), 2010. [documento da internet] [acessado $2013 \mathrm{dez} 11$ ]. Disponível em: http://www.climasaude.icict.fiocruz.br/ docs/vulnerabilidade_manaus_relat_final2_x_edit.pdf

19. Brasil. Ministério da Integração Nacional. Secretaria Nacional de Defesa Civil. Centro Nacional de Gerenciamento de Riscos e Desastres (Cenad). Anuário brasileiro de desastres naturais: 2012. Brasília: Cenad; 2013.

20. Articulação Semiárido Brasileiro (ASA). Semiárido [documento da internet]. 2013 [acessado $2013 \mathrm{dez}$ 12], [4 p.]. Disponível em: http://www.asabrasil.org.br/Portal/ Informacoes.asp?COD_MENU $=105$

21. Programa das Nações Unidas para o Desenvolvimento (PNUD). 70\% das cidades de menor IDH são áridas - Dos mil municípios com menor Índice de Desenvolvimento Humano, 715 ficam em região de alta aridez; 915 estão pior que a Namíbia. [Notícias PNUD - documento da internet]. 2006 [acessado $2013 \mathrm{dez} 12$ ]; [2 p.]. Disponível em: http://www.pnud.org.br/Noticia. aspx?id $=3206$

22. Instituto Brasileiro de Geografia e Estatística (IBGE). Censo Demográfico 2010: Aglomerados Subnormais: primeiros resultados. Rio de Janeiro: IBGE; 2010.

23. Castellanos PL. Epidemiologia, saúde pública, situação de saúde e condições de vida. Considerações conceituais. In: Barata RB, organizadora. Condições de Vida e Situação de Saúde. Rio de Janeiro: Abrasco; 1997. p. 31-75.

Artigo apresentado em 06/03/2014

Aprovado em 06/06/2014

Versão final apresentada em 08/06/2014 